\title{
Studies of the Agarose Gelling System by the Fluorescence Polarization Method. III.*
}

\author{
Akira HAYASHI, Kyosuke KINOSHITA, and Shiro YASUEDA \\ Department of Chemistry, Ritsumeikan University, \\ Tojiinkita-machi, Kita-ku, Kyoto 603, Japan.
}

(Received January 18, 1980)

\begin{abstract}
In order to confirm the gelling mechanism of agarose, the gel point, coil-helix point, gel melting point, and certain physical properties were measured at various concentrations below $5 \mathrm{~g} \mathrm{dl}^{-1}$. A phase diagram of the gelling system was obtained by plotting the reciprocals of these transition temperatures and the changing temperatures of the physical properties against the concentration. This diagram is basically quite similar to that for the rod-like polymer systems proposed by Flory. From the qualitative coincidence of both diagrams, it was confirmed that gelation was caused by the phase separation of the helical segment into isotropic and anisotropic solution phases. Characteristic properties of the gel such as turbidity, electric conductivity, specific optical rotation, pore size, and swelling were interpreted by the gel structure based on the gelling mechanism and by the gel composition based on the phase diagram.
\end{abstract}

KEY WORDS Agarose / Gelling Mechanism / Coil-Helix Transition / Phase Separation / Rod-like Polymer / Optical Rotation of Gel / Swelling of Gel /

Agarose is basically a regular polymer composed of repeating units of agarobiose, (1-4)-3,6anhydro-L-galactosyl-(1-3)-D-galactose. This regular polymer chain folds into a helix at low temperatures if there are no substituted groups. Some of the repeating units in the molecule are substituted by charged groups, and these units cannot fold into the helical chain owing to steric hinderance. Thus, the segment neighboring with the substituted unit is always flexible in solution and is called the kink segment. The agarose macromolecule is thus composed of an alternating chain of the helix segment with the kink segment, and is a rigid but flexible molecule as a whole in solution. ${ }^{1}$

The gelation of agarose is related to the helix formation of the molecular chain. According to Rees et al., ${ }^{2}$ the junction zones of the network are caused by the formation of intermolecular double helices in solution. These helices aggregate into the boundles, which constitute the framework of the network. Consequently, the gel point coincides with

* Presented at SDSJ 28th Symposium on Macromolecules, the Society of Polymer Science, Osaka, December 1-3, 1979; Polym. Prepr., Jpn., 28 (7), 1414 (1979). Part II of the series is ref 4. the helix point according to the proposal of these authors.

The authors have studied the gelling process of agarose by the fluorescence polarization method and found that the coil-helix transition point existed independently against the gel point. ${ }^{3}$ The helices made at the helix point should be intramolecular in the dilute solution such as $0.3 \mathrm{~g} \mathrm{dl}^{-1}$ and should not develop alone into the junction of network. Also, the helix formation proposed by Rees, must be studied more to come to an understanding about the formation of the junction zone. With further studies on the gelling system, ${ }^{4}$ the generation of small droplet could be observed in a dilute solution at the gel point and the gel point was proposed as the phase separation point of the system into the dilute isotropic and the concentrated anisotropic solutions, according to Flory's theory. ${ }^{5}$ This proposal means that the junction zone is the droplet of the anisopropic phase, coacervate, and that the framework is the segment dissolved in the isotropic phase.

Flory's theory of phase equilibrium in rod-like polymer solutions is summarized by the phase diagram in Figure 1. In this figure, $\chi_{1}$ is the 
interaction parameter and $v_{2}$ is the volume fraction of rod-like molecule. $v_{2}^{\prime}$ and $v_{2}^{\prime \prime}$ are the upper limit concentration of the isotropic phase and the lower limit of the anisotropic phase, respectively.

The gelation of agarose occurs very quickly and the principal driving factor should be the behavior of the helical segment in the agarose molecule. It is expected that the helical segment behaves like the isolated rod-like molecule, though some restriction of the motion should occur under a circumstance such that the non-helical kink segment co-exists. If the authors' proposal are correct, the gel point of agarose solutions should obey Flory's characteristic phase diagram in Figure 1. In this case, the rod-like molecule must be replaced by the rod-like helical segment in the agarose macromolecule.

In the experiment with agarose, it is expected that the interaction parameter, $\chi_{1}$, is in proportion to the reciprocal of temperature since the gelling system has a clear, upper critical solution temperature. Therefore, the relation of the reciprocal of the gel point to $v_{2}$ is also expected to resemble the diagram in Figure 1.

It should be recalled here that the agarose gelling system shows a hysteresis phenomenone. Rod-like helical segments tend to aggregate into a threedimensional crystalline phase even in solution. The melting point of such a crystalline phase is higher than that of the original helix, one-dimensional crystal. This is the reason for the hysteresis in agarose gelling system and thus the gel point and the coil-helix transition point are not the exact equilibrium points. However, the rate of crystallization is expected to be fairly slow in dilute solution systems because the crystallization of high polymer is usually nucleation controlled and also because the

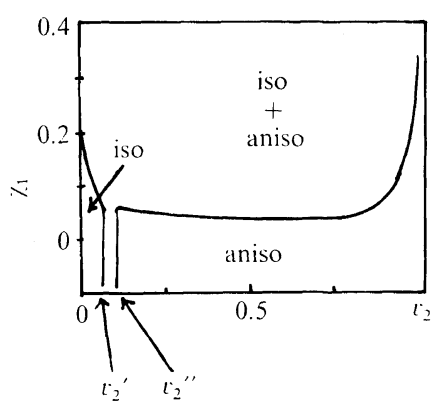

Figure 1. Phase diagram for a rod-like polymer system with axial ratio $x=150$ in solution by Flory. ${ }^{5}$ gel point of agarose is close to the helix point. In this paper, the effect of hysteresis, therefore, was neglected when the gel point was measured.

The measurements of the coil-helix transition point $\left(T_{2}\right)$, and the melting point $\left(T_{3}\right)$ were carried out in addition to that of the gel point $\left(T_{1}\right)$, so as to clarify the whole transformation diagram of the agarose system.

\section{EXPERIMENTAL}

The sample of agarose used was "Agarose A-37" (Nakarai Chemicals Ltd., Kyoto, Japan, Lot M5H 4652). The upper limit of the agarose concentration was $5 \mathrm{~g} \mathrm{dl}^{-1}$ owing to the difficulty of preparating homogeneous, more concentrated solutions. Within the concentration range, the most characteristic part of the diagram in Figure 1 is included as shown later.

Figure 2 shows the diagram which is to show the expected experimental results. In this diagram, concentrations are presented in $\mathrm{g} \mathrm{dl}^{-1}$, instead of volume fraction: $c, c^{\prime}$, and $c^{\prime \prime}$ instead of $v_{2}, v_{2}{ }^{\prime}$, and $v_{2}^{\prime \prime}$, respectively. The experimental plan was made so that each line in the figure was checked by more than two different methods, except in the case of $T_{3}$. The vertical arrows in the figure represent the experiments in which the temperature varies at a constant concentration, and horizontal arrows show the experiments in which concentration varies at a constant temperature. Experimental methods are as following.

Fluorescence Polarization, $p$. The probe dye was uranine $\left(10^{-6} \mathrm{M}\right)$ and the measurement was the same as that in previous papers. ${ }^{3,4}$

Fluorescent Intensity, $I_{\mathrm{f}}$. The sample solution was the same as that for the fluorescence polarization. The fluorescence thus came from uranine and the

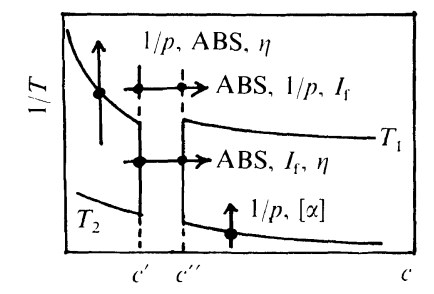

Figure 2. Experimental plans for checking the phase diagram. Experimental methods are described in the text. 
intensity change observed was due mainly to the change in the turbidity of the solution.

Optical Absorption, ABS. A Hitachi 100-50 Spectrophotometer was used and the wavelength at which uranine had no absorption was $540 \mathrm{~nm}$. The purpose for measuring the $A B S$ was to check the turbidity change in the solution.

Viscosity, $\eta$. The gel point $\left(T_{1}\right)$ and melting point $\left(T_{3}\right)$ were estimated according to the behavior of the steel ball $(\phi=2 \mathrm{~mm})$ placed on the surface of the solution. The steel ball stopped sinking in the solution at the gel point, and started to sink again at the melting point. For the determining of $c^{\prime}$ and $c^{\prime \prime}$, change of viscosity was used; the steel ball $(\phi=0.8 \mathrm{~mm})$ was fallen from the surface of the solution and the falling time corresponding to a certain distance in the solution was measured. The reduced viscosities at the temperatures above $T_{2}$ were measured using an Ubbelohde-type viscometer.

Specific Optical Rotation, $[\alpha]$. An Union Automatic Degital Polarimeter PM-101 was used. The wavelength was $405 \mathrm{~nm}$. The cell length was $10 \mathrm{~cm}$ for the solution and $1 \mathrm{~cm}$ for the gel.

\section{RESULTS}

\section{Transition Point Measurements}

Gel Point $\left(T_{1}\right)$. Estimation of $T_{1}$ by fluorescence polarization was described in a previous paper. ${ }^{3}$ From the $A B S, T_{1}$ was estimated as the starting temperature of the turbidity. Figure 3 shows the $T_{1}$ vs. $c$ relationships estimated by $1 / p, A B S$, and $\eta$. There seems to be some scattering, depending upon the method used, but the inclinations of the curves

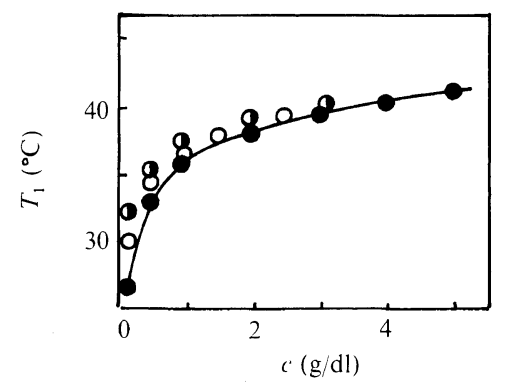

Figure 3. Relation of gel point $\left(T_{1}\right)$ to concentration with optical absorption ( $)$, fluorescence polarization $(\mathrm{O})$, and viscosity are quite similar. For the final plots in Figure 11, the results from the viscosity were used.

The gel point of "Agarose A-37" changed to some extent from one lot to another. The $T_{1}$ of this experiment was a little lower than that in previous papers. $^{3,4}$

Coil-Helix Transition Point $\left(T_{2}\right)$. The existence of $T_{2}$, besides $T_{1}$, was found by the fluorescence polarization method. ${ }^{3}$ Estimation of $T_{2}$ from $[\alpha]$ was somewhat difficult as will be described later. Determination of $T_{2}$ by $[\alpha]$, however, could be carried out up to a concentration of $5 \mathrm{~g} \mathrm{dl}^{-1}$.

Figure 4 shows the results. Similar inclinations were obtained by both methods.

Melting Point $\left(T_{3}\right)$. The results from $\eta$ are shown in Figure 5.

Measurements of the Upper Limit Concentration of the Isotropic Phase ( $\left.c^{\prime}\right)$ and the Lower Limit of the Anisotropic Phase $\left(c^{\prime \prime}\right)$

Temperatures below Gel Point $\left(T_{1}\right)$. The $I_{\mathrm{f}}, p$, and $A B S$ of the agarose gel containing uranine were measured at room temperature, in order to estimate $c^{\prime}$ and $c^{\prime \prime}$. Figure 6 shows the relation of each

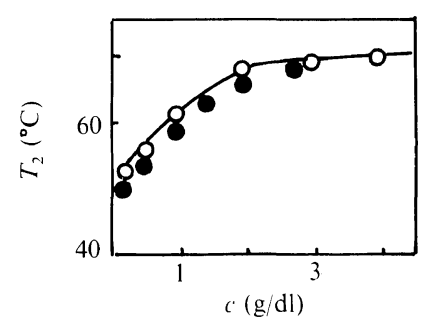

Figure 4. Relation of helix point $\left(T_{2}\right)$ to concentration with optical rotation $(O)$ and fluorescence polarization (O).

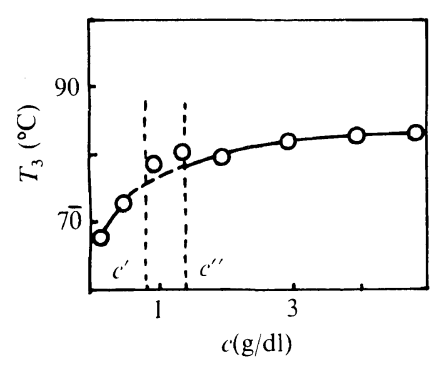

Figure 5. Relation of gel melting point $\left(T_{3}\right)$ to concentration with viscosity. 


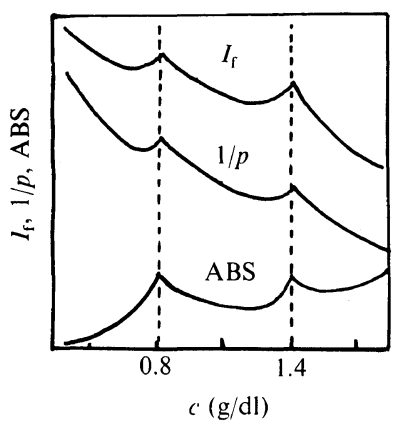

Figure 6. Changes in fluorescent intensity $\left(I_{\mathrm{f}}\right)$, fluorescence polarization $(1 / p)$, and optical absorption $(A B S)$ with gel concentration.

measured value (arbitrary scale) to the concentration. All three curves have similar inflexion points at the same concentrations, $0.8 \mathrm{~g} \mathrm{dl}^{-1}$ and 1.4 $\mathrm{g} \mathrm{dl}^{-1}$. These concentrations may correspond to $c^{\prime}$ and $c^{\prime \prime}$. The data obtained in the concentration range from $c^{\prime}$ to $c^{\prime \prime}$ were unstable and lacking in reproducibility. However, detection of $c^{\prime}$ and $c^{\prime \prime}$ was easily estimated and reproducible. The unstability of the data in this concentration range could always be observed in Figures 3-7, and 10. Solutions in the range from $c^{\prime}$ to $c^{\prime \prime}$ were already separated into two phases before reaching $T_{1}$; considerable time was required for the separation reaction to equilibrate completely since the rod-like segments constitute only a part of the flexible macromolecule. The unstability caused by the delay of the equilibrium certainly influences the physical properties of the gel at temperatures below $T_{1}$.

Temperatures between $T_{1}$ and $T_{2}$. Figure 7 shows the falling time of the steel ball $(\phi=0.8 \mathrm{~mm})$ at $45^{\circ} \mathrm{C}$ and $49^{\circ} \mathrm{C}$. The limit concentrations, $c^{\prime}$ and $c^{\prime \prime}$, are determined by this time as 0.8 and $1.4 \mathrm{~g} \mathrm{dl}^{-1}$, as same as those at the temperatures below $T_{1}$ in Figure 6. By the phase separation, a three-dimensional network is formed in the solution in that the agarose macromolecule penetrates through both phases. Therefore, the viscosity becomes much higher in this concentration range.

Measurements of $I_{\mathrm{f}}$ and $A B S$ were not so useful for detecting the $c^{\prime}$ and $c^{\prime \prime}$, because changes in these values of them were very slight at these concentrations.

Temperatures above $T_{2}$. Figure 8 shows the relation of reduced viscosity to the concentration at $72^{\circ} \mathrm{C}$. There seems to be no inflexion point

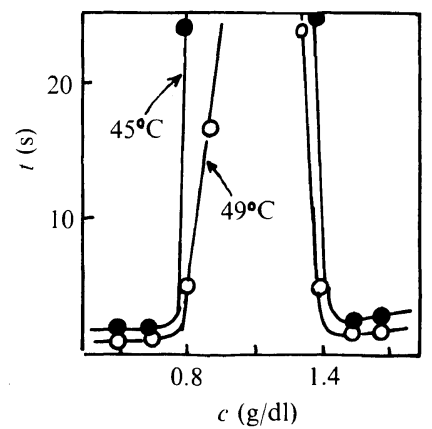

Figure 7. Changes in the falling time of the steel ball $(\phi=0.8 \mathrm{~mm})$ in solution with concentration, at $45^{\circ} \mathrm{C}$ and $49^{\circ} \mathrm{C}(\mathrm{O})$.

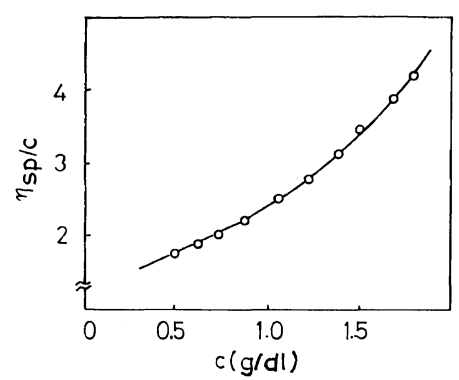

Figure 8. Relation of reduced viscosity to concentration at $72^{\circ} \mathrm{C}$.

corresponding to $c^{\prime}$ or $c^{\prime \prime}$, meaning that the agarose macromolecule has the form of a random coil at temperatures above $T_{2}$. This result is also strong evidence of $T_{2}$ 's being a coil-helix transition point (compare Figure 8 with Figure 7).

\section{Specific Optical Rotation of Agarose Solutions}

Figure 9 shows the relation of $[\alpha]$ to the temperature in the course of the gelling process (at $0.6 \mathrm{~g} \mathrm{dl}^{-1}$ ). The detection of $T_{2}$ is somewhat difficult, though the inclination of the coil state is a little more than that of the helical state. A small peak was sometimes observed at $T_{2}$, especially at lower concentrations, as shown in the figure, but no peak at concentrations higher than $1 \mathrm{gdl}^{-1}$ could be observed.

$[\alpha]$ at constant temperature is substantially constant with changes in concentrations for cases of the helical and coil stages. The specific optical rotation of the gel, $[\alpha]_{\text {gel }}$, however, negatively increases 
Studies of Agarose Gel by Fluorescence Method. III.

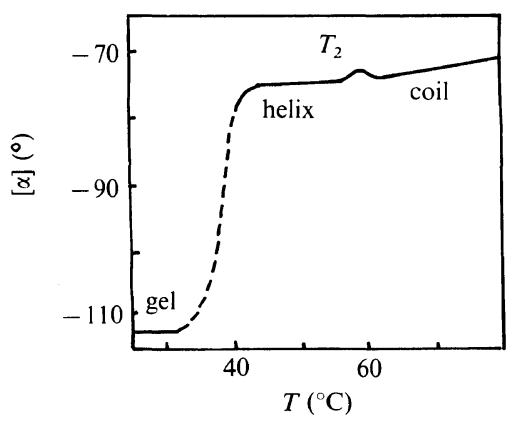

Figure 9. Relation of specific optical rotation to temperature at $0.6 \mathrm{~g} \mathrm{dl}^{-1}$.

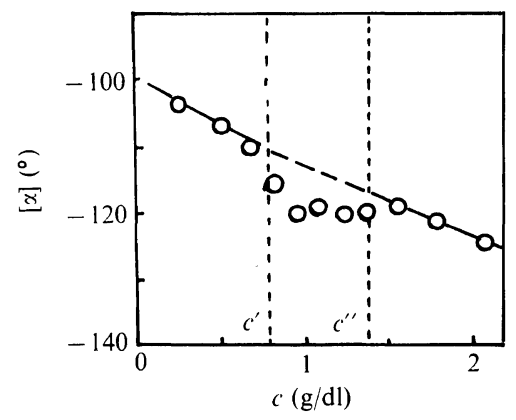

Figure 10. Relation of specific optical rotation of gel to concentration.

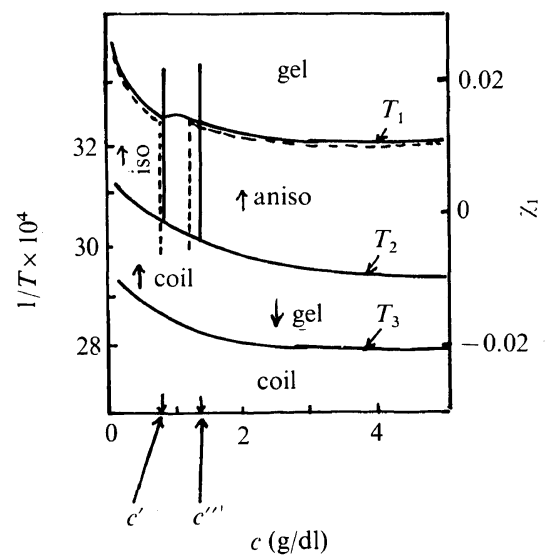

Figure 11. Whole phase diagram of agarose gelling system (-) and calculated line (----).

linearly with an increase in concentration as shown in Figure 10. There being no remarkable difference in the $[\alpha]$ values for the helical and coil states was an unexpected result. The authors have no data and no ideas on which to base further discussion on the $[\alpha]$ of the helical and coil states. $[\alpha]_{\text {gel }}$ will be discussed later.

Phase Diagram of Agarose Transformation from Solution to Gel and its Reverse

Figure 11 shows the relation of the reciprocal of the temperature in Figures $3-7$ to the concentration, and shows the phase diagram of the agarose transformation from the solution to gel and its reverse. The dotted line in the figure is the caluculated values described later.

\section{DISCUSSION}

It is seen that the upper part of the Figure 11 basically resembles the lower concentration part of the Figure 1. In particular, it is interesting that the characteristic concentration limits, $c^{\prime}$ and $c^{\prime \prime}$, are clearly found in the diagram. The gelation of agarose is caused by the phase separation of the rodlike helical segment in the molecule as the authors have proposed.

The density of agarose could be estimated as about 1.5 , using an analogy with related polysaccharides. An approximate volume fraction is, therefore, $\quad v_{2} \cong c / 1.5$ at lower concentrations. However, the values of $c^{\prime}$ and $c^{\prime \prime}$ in Figure 11 seem too small compared with those in Figure 1. The rodlike hlical segment considered here is not an isolated molecule but only a rod-like part in the flexible macromolecule as a whole. Thus making the kinetics of the rod-like segment is rather complex. Consequently, $c^{\prime}$ and $c^{\prime \prime}$ cannot be discussed quantitatively here.

As an approximate calculation, $v_{2}{ }^{\prime} \cong c^{\prime}=0.008$ and $v_{2}^{\prime \prime} \cong c^{\prime \prime}=0.014$ have been used without any correction in this paper. Since the relations of the limit concentrations to the axial ratio of rod-like chain, $x$, are expressed by $v_{2}{ }^{\prime}=8 / x$ and $v_{2}{ }^{\prime \prime}=12.5 / x,{ }^{5}$ the axial ratios were calculated as. $x=1,000$ and 900 for $v_{2}{ }^{\prime}$ and $v_{2}{ }^{\prime \prime}$, respectively. These values seem to coincide very well, because it is known that the larger the difference between $v_{2}{ }^{\prime}$ and $v_{2}{ }^{\prime \prime}$ becomes, the larger is the distribution of molecular weight. ${ }^{5}$ Too large a value, $x=1,000$, comes about for the same reason as that involved in the case of small $c^{\prime}$ and $c^{\prime \prime}$. 
From $x=1,000$, the relation of $\chi_{1}$ to $c$ at the phase separation point was calculated according to Flory's equations. ${ }^{5}$ The resulting line is superposed as a dotted line on the experimental data in Figure 11. The $\chi_{1}$ is presented in the right hand vertical axis. Both lines are well superimposable.

It is interesting that the physical properties of the gel in the concentration range from $c^{\prime}$ to $c^{\prime \prime}$ are somewhat different from those in other concentration ranges as shown by Figure 6 . This brings to mind the $c_{3}$ concentration in the previous paper. ${ }^{3}$ The $c_{3}$ was the concentration at which a certain structural change in gel was supposed to have occured by the sudden changes in $1 / p^{3}$ and elasticity. ${ }^{6}$ The $c_{3}$ point should correspond to the $c^{\prime}$ or $c^{\prime \prime}$ in this paper.

The phase diagram in Figure 11 shows the whole process of the agarose transformation between the solution and gel: during the gelling process from the hot solution, the system consists of an isotropic coil solution at temperatures above $T_{2}$, an isotropic helical solution $\left(<c^{\prime}\right)$, an anisotropic helical solution $\left(>c^{\prime \prime}\right)$, or a mixed heterogeneous solution of both $\left(c^{\prime}-c^{\prime \prime}\right)$ in the temperature range from $T_{2}$ to $T_{1}$, and the gel of the mixed heterogeneous solution at temperatures below $T_{1}$. During the melting process of the gel, the mixed phase of isotropic and anisotropic helical solutions does not change up to $T_{3}$, since the crystallization of helical segments occurs in the anisotropic phase and the melting point of the crystal corresponds to $T_{3}$. At $T_{3}$, the gel melts into the isotropic coil solution phase.

From the above discussion, the agarose gelling process is drawn as in Figure 12. The helical chain in agarose is a parallel double folding structure according to Rees. ${ }^{5}$ In the dilute solution, the intramolecular helix should predominate over the intermolecular one. Motions of the helical segment should be different from the isolated rod-like

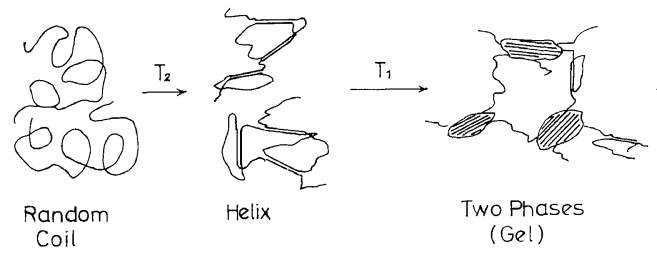

Figure 12. Schematic representation of agarose gelling process. molecule dealt with by Flory. However, the motion basically obeys Flory's theory as shown in Figure 11. The phase separation of the helical chains is thus the driving factor for the gelation at $T_{1}$, though the neighbored kink segments are still in a random coil state. In the gel state, the agarose macromolecule penetrates through both solution phases. The junction zone of the network is the droplet of anisotropic phase, and the framework is the agarose segment, consisting of the helical and kink segments, dissolved in the dilute isotropic solution phase.

Figure 13 shows the composition of the both phases in gel. At $T^{\circ} \mathrm{C}$, the gel $\mathrm{X}$ of $c_{\mathrm{x}} \mathrm{g} \mathrm{dl}^{-1}$ consists of the isotropic phase $\mathrm{A}$ of $c_{\mathrm{A}} \mathrm{g} \mathrm{dl}^{-1}$ with the anisotropic phase $\mathrm{B}$ of $c_{\mathrm{B}} \mathrm{g} \mathrm{dl}^{-1}$. The ratio of the two phases is $x_{\text {iso }}: x_{\text {aniso, }}$, where $x_{\text {iso }}+x_{\text {aniso }}=1$. It must be remembered that the $c_{\mathrm{A}}$ and $c_{\mathrm{B}}$ do not changed with a change in $c_{\mathrm{x}}$ provided the temperature is constant. From the calculations made at room temperature, $c_{\mathrm{B}}$ is extreamely high; $c_{\mathrm{B}} \cong 0.9$ (as volume fraction, $\left.v_{2}\right)$. At such a high concentration, agarose helices are highly oriented and are in the liquid-crystalline state. By the polarizing microscope, the existence of such an oriented droplet of the phase $B$ is observable in the dilute gel. An increase in the orientation in the droplet could be also observable after several days and this must be the result of an increase in the degree of crystallization in the phase $B$.

From the gel structure and composition shown by Figures 12 and 13, some of the characteristic properties of agarose gel are properly interpreted as following.

(a) The gelation of agarose occurs very quickly because the driving factor of the reaction is the

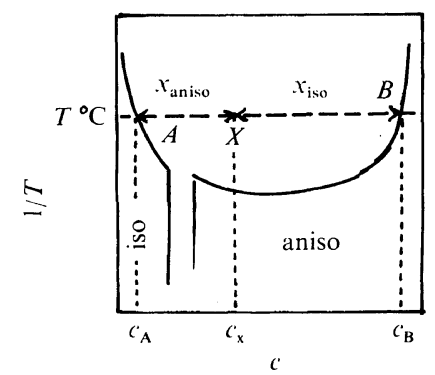

Figure 13. Schematic representation of gel composition. 
phase separation, or a kind of phase transition. The gelation of other polymers such as gelatin or PVA, in which the reaction proceeds very slowly, should be a kinetically controlled reaction such as crystallization. The gelation of agarose follows a sudden turbidity increase at the same time because of the development of heterogeneous solution in phase $\mathrm{A}$ with B.

(b) The electric conductivity of the gel is composed basically only of that of the phase A, since most of the gel is occupied by the phase A. $c_{\mathrm{A}}$ does not change with the change in the $c_{\mathrm{x}}$ as mentioned above, and the electric conductivity is thus substantially unchangeable in the lower concentration range.

(c) The optical rotation of the gel is the sum of rotations of the phases A and B. The specific optical rotation of the gel of $c_{\mathrm{x}}$ is given by the following equation.

$$
[\alpha]_{\mathrm{gel}}^{c_{\mathrm{x}}}=x_{\text {iso }}[\alpha]_{\text {iso }}^{c_{\mathrm{A}}}+x_{\text {aniso }}[\alpha]_{\text {aniso }}^{c_{\mathrm{B}}}
$$

where $[\alpha]_{\text {iso }}^{c_{A}}$ and $[\alpha]_{\text {aniso }}^{c_{B}}$ are the specific optical rotations of the phase A and $B$, respectively. It is expected that the $[\alpha]_{a n i s o}^{c \mathrm{~B}}$ contains an additional rotation based on the orientation effect in addition to $[\alpha]_{\text {iso. }}^{c_{A}}$. If the $[\alpha]_{\text {aniso }}^{c_{B}}$ is negatively larger than the $[\alpha]_{\text {iso }}^{c} \mathrm{~A}$ the relation of $[\alpha]_{\mathrm{gel}}^{c_{\mathrm{x}}}$ to $c_{\mathrm{x}}$ is given by Figure 14 . This figure provides a good explanation for the experimental data in Figure 10.

(d) The large pore size of the agarose gel membrane is easily explained by the gel structure and composition in Figures 12 and 13.

(e) If an agarose gel is placed in water at a

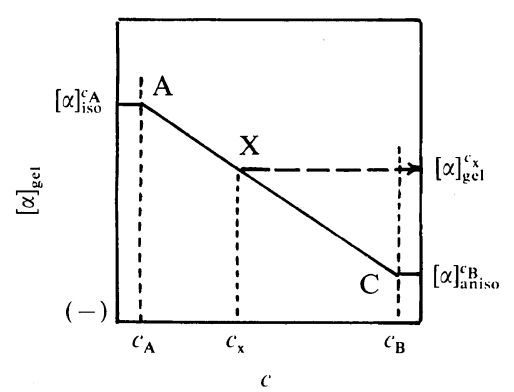

Figure 14. Schematic representation of optical rotation of gel.

constant temperature below $T_{1}$, it is expected that phase $\mathrm{B}$ is transformed into phase $\mathrm{A}$, thus causing an expansion in the volume since neither $c_{\mathrm{A}}$ nor $c_{\mathrm{B}}$ can change. However, the transformation from phase $\mathrm{B}$ to $\mathrm{A}$ is practically difficult, owing to the formation of a crystalline phase in phase $\mathrm{B}$, and the gel is unable to swell beyond the initial volume.

\section{REFERENCES}

1. D. A. Rees, Adv. Carbohyd. Chem., 24, 267 (1969).

2. S. Arnott, A. Fulmer, W. E. Scott, I. C. M. Dea, R. Moorhouse, and D. A. Rees, J. Mol. Biol., 90, 269 (1974).

3. A. Hayashi, K. Kinoshita, and M. Kuwano, Polym. J., 9, 219 (1977).

4. A. Hayashi, K. Kinoshita, M. Kuwano, and A. Nose, Polym. J., 10, 485 (1978).

5. P. J. Flory, Proc. R. Soc. London, Ser. A, 234, 73 (1956).

6. N. Hirai, Nippon Kagaku Zasshi, 72, 837 (1951). 